\title{
Are Auditory Hallucinations Related to the Brain's Resting State Activity? A 'Neurophenomenal Resting State Hypothesis'
}

\author{
Georg Northoff \\ Institute of Mental Health Research, University of Ottawa, Ottawa, Canada, Taipei Medical University, Graduate Institute of Humanities \\ in Medicine, Taipei, Taiwan, Taipei Medical University-Shuang Ho Hospital, Brain and Consciousness Research Center, New Taipei City, \\ Taiwan, National Chengchi University, Research Center for Mind, Brain and Learning, Taipei, Taiwan, National Chengchi University, \\ Department of Psychology, Taipei, Taiwan, Centre for Cognition and Brain Disorders (CBBD), Normal University Hangzhou, Hangzhou, China
}

\begin{abstract}
While several hypotheses about the neural mechanisms underlying auditory verbal hallucinations (AVH) have been suggested, the exact role of the recently highlighted intrinsic resting state activity of the brain remains unclear. Based on recent findings, we therefore developed what we call the 'resting state hypotheses' of $\mathrm{AVH}$. Our hypothesis suggest that AVH may be traced back to abnormally elevated resting state activity in auditory cortex itself, abnormal modulation of the auditory cortex by anterior cortical midline regions as part of the default-mode network, and neural confusion between auditory cortical resting state changes and stimulus-induced activity. We discuss evidence in favour of our 'resting state hypothesis' and show its correspondence with phenomenal, i.e., subjective-experiential features as explored in phenomenological accounts. Therefore I speak of a 'neurophenomenal resting state hypothesis' of auditory hallucinations in schizophrenia.
\end{abstract}

KEY WORDS: Hallucinations; Schizophrenia; Intrinsic activity; Auditory cortex.

\section{INTRODUCTION}

Auditory verbal hallucinations (AVH) refer to the experience of perceiving speech in the auditory modality without corresponding external stimuli. Up to $60-80 \%$ of patients with schizophrenia suffer from AVH which can also occur in conditions other than schizophrenia as for instance in drug-induced psychosis or even healthy subjects. This has let to several hypothesis including cognitive-based top-down, sensory-based bottom-up, ${ }^{1-3)}$ combined bottom-up and top-down models, ${ }^{4-6)}$ and social ${ }^{7,8)}$ hypotheses about the neural mechanisms underlying AVH.

Recent investigations point out the crucial relevance of the brain's resting state activity in various networks of the brain including the so-called default-mode network (DMN). The DMN includes various regions like the anterior and posterior cortical midline structures (CMS) as

Received: April 14, 2014 / Revised: June 19, 2014

Accepted: June 23, 2014

Address for correspondence: Georg Northoff, MD, PhD Research Unit Director, Mind, Brain Imaging and Neuroethics Institute of Mental Health Research (IMHR), 1145 Carling Avenue, Ottawa, ON K1Z 7K4, Canada

Tel: +1-613-7226521 ext. 6870, Fax: +1-613-7226521 (ext 6801) E-mail: georg.northoff@theroyal.ca well as the lateral parietal cortex and the hippocampus. ${ }^{9,10}$ While the resting state activity level seems to be particularly high in the DMN, its exact impact on stimulus-induced activity remains unclear though. ${ }^{11)}$ Most interestingly, resting state activity in DMN may not only be relevant neuronally but also for psychological and phenomenal functions like self and consciousness. ${ }^{12,13)}$ This transforms a purely neuronal approach to DMN and resting state activity into what can be called a neurophenomenal approach. $^{12,13)}$

However, resting state activity is not limited to the DMN and its midline regions but can be found within all regions and networks throughout the whole brain including in auditory cortex. ${ }^{14)}$ This is relevant since patients with AVH have been observed to show abnormally high resting state activity in auditory cortex before or during the onset of their hallucinations. ${ }^{15)}$ This raises two questions. First, how does the abnormally increased resting state activity in auditory cortex impact subsequent stimulus-induced activity and auditory perception? Second, what is the origin of the apparently abnormally increased resting state activity in auditory cortex during $\mathrm{AVH}-$ could it be related to resting state activity changes in other regions as for instance the DMN?

(c) This is an Open-Access article distributed under the terms of the Creative Commons Attribution Non-Commercial License (http://creativecommons.org/licenses/by-nc/3.0) which permits unrestricted non-commercial use, distribution, and reproduction in any medium, provided the original work is properly cited. 
The general aim of this paper is to develop a neural hypothesis that focuses specifically on the possible role of the brain's resting state activity in generating AVH, the 'resting state hypothesis of AVH'. More specifically, I aim to link some of the neural features of the brain's auditory cortical resting state activity in schizophrenia to auditory hallucinations and some of their phenomenal, i.e., subjective-experiential, features as discussed in phenomenological psychiatry. Therefore I speak of a 'neurophenomenal resting state hypothesis' (see title). We will develop such resting state hypothesis in three steps, abnormal resting state activity in auditory cortex, abnormal rest-rest interaction between auditory cortex and DMN, and reduced rest-stimulus interaction in auditory cortex. In contrast, we here neglect alternative theories of auditory hallucination (sensory-based bottom-up and cognitive-based topdown approaches) for which we refer to the literature. ${ }^{16-19)}$

\section{MAIN SUBJECTS}

\section{Resting State Hypothesis of AVH I: Abnormally Increased Resting State Activity in the Auditory Cortex}

Early functional studies observed an association of hallucinations/delusions as reality distortions with increased resting state activity (e.g., metabolism or perfusion) in the superior or middle temporal lobe that includes the auditory cortex. ${ }^{20,21)}$ Comparing the resting state activity during AVH with the one during the absence of AVH within the same patients, observed hallucination-related activity in Broca's area, the temporal gyri and the primary auditory cortex. ${ }^{15)}$ Taken together, these findings lend support to the assumption that especially the secondary (but also the primary) auditory cortex may show increased resting state activity in the acute psychotic state when suffering from auditory hallucinations.

More recent investigations observed too increased resting state activity in auditory cortex. For instance, increased cerebral blood flow in superior temporal gyrus including the auditory cortex was observed to be remain stable over time in patients with schizophrenia with treatment-resistant $\mathrm{AVH}^{2}{ }^{22)}$ Resting state functional connectivity from right and left superior temporal gyrus (and parahippocampal region and inferior frontal cortex) was observed to be increased in non-psychotic individuals with AVH when compared to non-hallucination subjects $^{23)}$; see also the reference ${ }^{24)}$ for resting state functional connectivity in psychotic patients with AVH. Most interestingly, resting state functional connectivity from auditory cortex to speech related areas like the inferior frontal
A

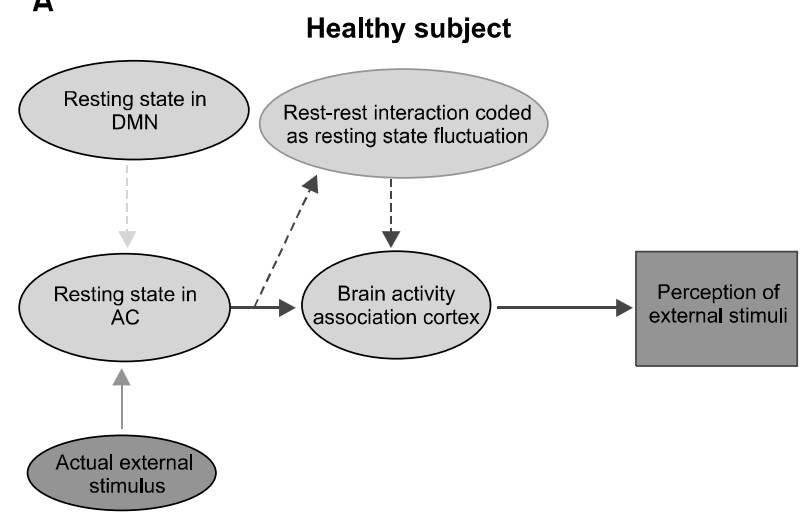

B
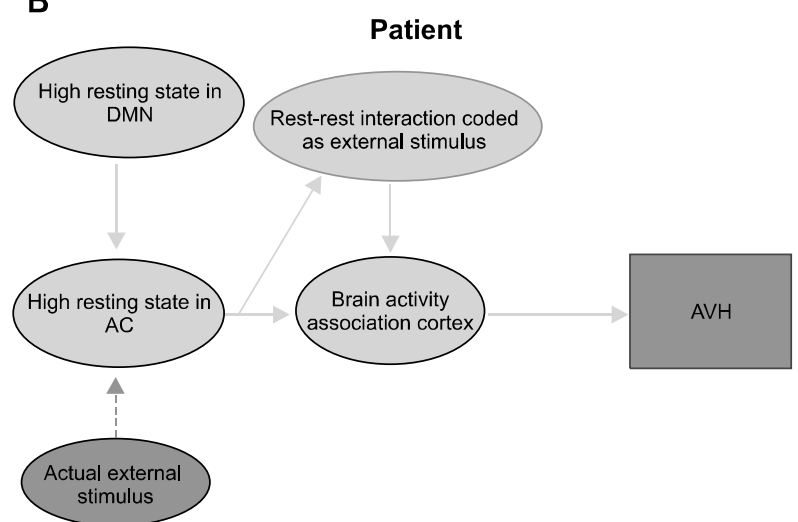

Fig. 1. This figure schematically illustrates our resting state hypothesis of the auditory verbal hallucination (AVH). (A) Healthy subjects. We hypothesize that the external stimuli can induce neural activity in the and related association cortex. At the same time, the resting state activity in the auditory cortex (AC) and other brain regions can interact with each other which the resulting neural difference from such rest-rest interaction being smaller than the one between resting state and stimulus-induced activity, e.g., rest-stimulus interaction. This larger neural difference during rest-stimulus interaction is supposed to generate the perception of the external stimuli. The broken line represents the smaller neural difference in the resting state itself, e.g., rest-rest interaction, when compared to the one between resting state and stimulus-induced activity from the external stimuli, e.g., rest-stimulus interaction. (B) Patients with $\mathrm{AVH}$. We hypothesize that high resting state in default-mode network (DMN) induces high resting state in $\mathrm{AC}$. This leads to high and increased larger neural differences during rest-rest interaction in the $\mathrm{AC}$ and subsequent auditory pathways. At the same time though such increased rest-rest interaction with larger neural differences diminishes the impact of external stimuli on neural activity in the AC thus leading to decreased rest-stimulus interaction. Rest-rest interaction is thus confused with rest-stimulus interaction which in turn leads to the generation of $\mathrm{AVH}$. The broken line represents the larger neural difference in the resting state itself, e.g., increased rest-rest interaction, when compared to the one between resting state and stimulus-induced activity from the external stimuli, e.g., rest-stimulus interaction.

Dark gray indicates the effect of external stimuli, e.g., rest-stimulus interaction, while light gray describes the effects within the resting state itself, e.g., rest-rest interaction. 
cortex was increased and correlated with the severity of AVH in patients with schizophrenia. ${ }^{25,26)}$

Taken together, there is solid evidence of abnormally elevated resting state activity in the auditory cortex itself. This though raises two questions. First, where does this abnormal increase in auditory cortical resting state activity come from? Second, how does it affect the neural processing of exteroceptive stimuli so that even in the absence of the latter external voices are heard. These issues will be discussed in the next sections.

\section{Resting State Hypothesis II: Elevated Resting State Activity in the Default-mode Network and Altered Rest-rest Interaction}

Recent imaging studies in schizophrenia reported indeed abnormal resting state activity and connectivity in the DMN. One study demonstrated that the medial prefrontal cortex and the posterior cingulate cortex/precuneus show decreased task-induced deactivation (TID) during a working memory task in both patients with schizophrenia and their relatives when compared to healthy subjects. ${ }^{27)}$ This is indicative of decreased task-related suppression and possibly increased resting state activity. Furthermore, the very same patients with schizophrenia also showed increased connectivity of the anterior medial prefrontal cortical regions with the posterior regions, the posterior cingulate cortex. Both hyperconnectivity and decreased TID correlated negatively with each other meaning that the more decreased task-related suppression, the more increased the connectivity. Finally, both decreased TID and increased connectivity correlated with psychopathology, i.e., predominantly positive symptoms as measured with the positive and negative syndrome scale (PANSS). ${ }^{28)}$

Since these earlier studies, there have been numerous investigations of resting state functional connectivity in schizophrenia. Resting state functional connectivity in schizophrenia shows clear alterations which confirm the earlier assumption of schizophrenia being a "dysconnectivity syndrome" that affects the whole brain and its various networks: this is evidenced by decreased cross-network connectivity with reduction in the degree of small worldness, number of hubs, and modularity. ${ }^{29)}$ More specifically, resting state functional connectivity within the midline regions and the DMN tends to increase while lateral prefrontal cortical and sensorimotor (and cortico-subcortical) functional connectivity is rather decreased in schizophrenia. $^{27,29,30)}$ This is well compatible with the initial observation of stronger low frequency fluctuations in schizophrenia in especially the anterior midline regions. $^{28,31)}$

Based on these findings, one may for instance hypothesize that due to the DMN abnormalities, there may be abnormal rest-rest interaction between the DMN and the auditory cortex. If this hypothesis holds one would also predict that abnormal auditory cortical activity during AVH may be preceded by abnormal cross-regional rest-rest interaction with the DMN. Recent resting state studies observed indeed abnormal functional connectivity between auditory cortex and prefrontal midline (and lateral) regions which would lend some first though tentative support to the assumption of abnormal rest-rest interaction between DMN and auditory cortex. ${ }^{25,26,32)}$

In addition, one may also consider the lateral prefrontal regions which stand in an orthogonal relation to the anterior prefrontal DMN. ${ }^{33)}$ This orthogonal relationship between medial and lateral prefrontal cortex seems to be resolved in patients with schizophrenia with positive rather than negative relationship ${ }^{34)}$; this, as it may be assumed tentatively, may also affect the functional connectivity between DMN and auditory cortex (in though yet unclear ways).

How can such abnormal cross-regional rest-rest interaction between auditory cortex and DMN lead to the perception of external voices that are nevertheless perceived as relevant and personally important? The DMN and especially the CMS as core regions of the DMN are well known to be involved in processing the degree of self-relatedness or personal relevance of external stimuli. ${ }^{35-37)}$ Within this set of CMS one may distinguish between anterior and posterior CMS (aCMS, pCMS); the aCMS include the anterior cingulate cortex and the ventro- and dorsomedial prefrontal cortex while the pCMS encomprise the posterior cingulate, the medial parietal cortex, the precuneus and the retrosplenium. ${ }^{37)}$ Most interestingly, especially in aCMS a strong overlap between high resting state activity and self-related activity can be observed. ${ }^{37,38)}$

How does the involvement of the aCMS in self-relatedness can account for the often observed highly personalized, derogatory and second- or third-person nature of $\mathrm{AVH}$ ? Voices in AV are perceived as external voices and are nevertheless attributed an abnormally high degree of personal relevance, meaning or self-relatedness. How is this possible? In the same way any auditory stimulus of external origin is attributed a certain degree of self-relatedness when processed in aCMS, the internally generated auditory stimulus is also assigned self-relatedness and thus personal relevance. 
A

Healthy subjects

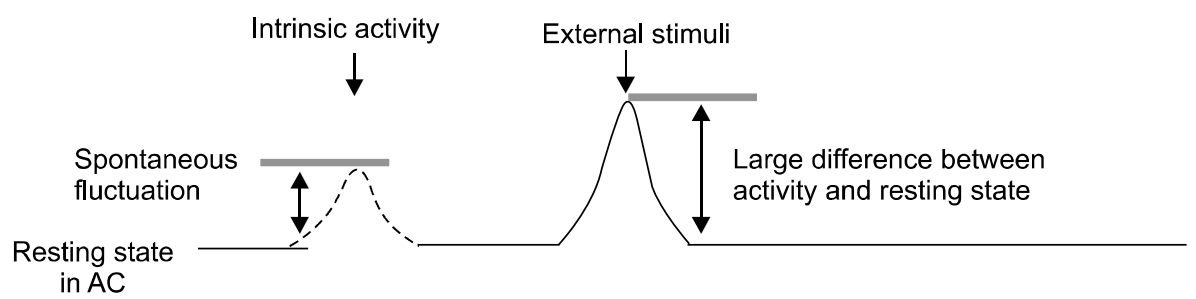

B

Patients with hallucinations

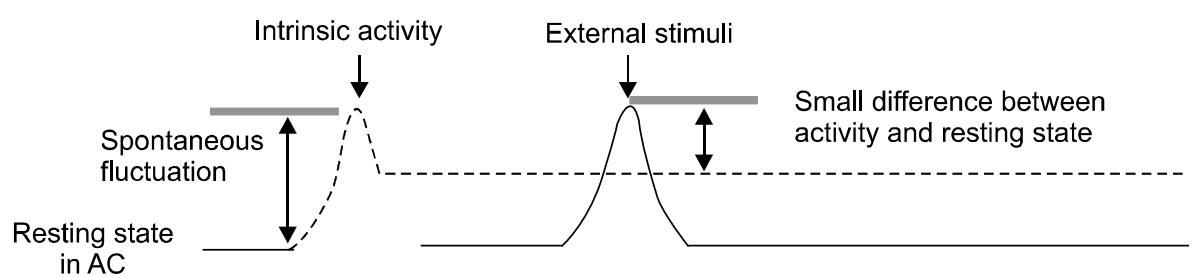

Fig. 2. This figure schematically illustrates the intrinsic activity, i.e., resting state activity and its fluctuations, in relation to extrinsic, i.e., stimulus-induced activity, in auditory cortex (AC) in healthy and patients with schizophrenia. (A) Healthy subjects. We hypothesize that intrinsic spontaneous fluctuation are not as large and are hence treated and coded as resting state fluctuations rather than as stimulus-induced activity. This enables the external stimuli to induce sufficient changes in neural activity, i.e., stimulus-induced activity, as distinct from the level of the resting state activity. (B) On the contrary, in patients with the auditory verbal hallucination (AVH), the intrinsic spontaneous fluctuation is either too large or/and does not return back to the original resting state activity level. The difference between original resting state level, i.e., before occurrence of the intrinsic fluctuation, and the resting state level after the fluctuation may then be as large as the change normally induced by external stimuli; the difference in activity level before and after the resting state fluctuation may then be coded by the brain as if it was induced by an external stimulus which in turn enables and predisposes to perceive it as AVH.

Moreover, since the internally auditory stimulus is derived from memory-based auditory experiences of previous external stimuli (see below for details), it may even be assigned an extremely high degree of personal relevance while still remaining in second- or third-person. Hence, we tentatively assume that the interaction between an internally generated stimulus in the auditory pathway and its subsequent neural processing in the hyperactive and hyperconnected aCMS may account for the highly personalized, derogatory and second- or third-person nature of AVH. The confusion between internal and external contents may thus be diminished which often is also described as reduced self-monitoring or self-recognition of internal contents as internal rather than external. ${ }^{19,39)}$

In sum, there is evidence for abnormal resting state functional connectivity in auditory cortex and its linkage to DMN and lateral frontal regions. Whether these abnormalities in resting state functional connectivity between auditory cortex and DMN (and lateral prefrontal cortex) can account for the abnormally high resting state activity as described above remains unclear at this point in time, however.

\section{Resting State Hypothesis of AVH III: Reduced} Rest-external Stimulus Interaction in the Auditory Cortex

What does the finding of a seemingly increased resting state activity in the auditory cortex imply for the neural processing of exteroceptive stimuli and hence for rest-stimulus interaction? The study by Ford et al. ${ }^{40)}$ suggest that there is decreased rest-stimulus interaction in auditory cortex in schizophrenia; this means that the external stimulus elicits less (or no) activity any more in the auditory cortex when compared to its resting state activity level. ${ }^{17,40)}$ Such reduced rest-(external) stimulus interaction leads no longer to the generation of a neural difference between resting state activity level and stimulus-induced activity; this may also account physiologically for the above described reductions in the amplitudes of early electrophysiological potentials during stimulus-induced activity. ${ }^{17,41)}$ This may reflect increased rest-rest interaction with increased processing of internal stimuli at the expense of external stimuli, e.g., rest-stimulus interaction.

If the onset of hallucinations is assumed to be triggered and preceded by abnormal rest-rest interaction in auditory cortex and subsequent regions of auditory processing, one would hypothesize abnormal neural activity in these re- 
gions to prior to the AVH. This has indeed been observed. Activity changes before the onset of AVH (i.e., 6-9s) were observed in the left inferior frontal cortex, the anterior cingulate cortex and the right middle temporal gyrus was evident 6-9s before the onset of AVH while during the AVH itself activity was present in the bilateral temporal gyrus. ${ }^{42-46)}$ There is also ample evidence for altered, i.e., reduced functional/effective connectivity from prefrontal regions like the dorsolateral prefrontal cortex (DLPFC), the anterior cingulate cortex and the left inferior frontal cortex to regions in the temporal cortex including the auditory cortex during verbal self-monitoring ${ }^{47)}$ or AVH themselves. $^{46,48)}$

In sum, there is ample evidence for abnormally strong rest-rest interaction in auditory cortex which, in consciousness on the level of experience, may be perceived as voices that come from external rather than internal (which psychologically is often described as deficit in self-monitoring or self-recognition [of internal contents]).

\section{Resting State Hypothesis IV: Convergence with Phenomenological Accounts - a Neurophenomenal Hypothesis}

Phenomenological approaches do not aim to explain neurobiological mechanisms in scientifically objective terms but on the personal significance and meaning of the patient's subjective experience, i.e., their phenomenal states. ${ }^{49-57)}$ This has led the phenomenological approaches to focus also on the self in patients with schizophrenia and their phenomenal states in the prior stages before the outbreak of acute symptoms, the subtle alterations in the subjective experience of self, other selves, and the world in future patients with schizophrenia ${ }^{52,58)}$ (see below for details). Hence, there has been exploration of the subjective experience of the self in the presymptomatic stages of schizophrenia of which the underlying neuronal mechanisms remains unclear though.

The phenomenological account focuses for instance on the subjective experience of the objective stimulus from the perspective of the experiencing person. It consecutively describes how we subjectively experience incoming sensory stimuli as exteroceptive auditory stimuli or interoceptive stimuli from our own body in a subjective way and thus how we can relate them to our own self. The linkage of sensory stimuli to the own self in subjective experience thus concerns what phenomenological accounts ${ }^{49,52,54,57-61)}$ describe as 'attunement'. The concept of 'attunement' describes, most broadly, the relation of the self to the world, e.g., how the self adjusts and adapts to the various objects, events and other persons in its respective environment.

Most specifically, phenomenologists point out that 'attunement' in this sense operates already on a prereflective, implicit or preconceptual level which Parnas ${ }^{52)}$ and Sass et al. ${ }^{58)}$ describe as preflective or preconceptual attunement. In the following I will use the concept of attunement to describe such pre-reflective, pre-conceptual and implicit adaptive processes which corresponds well to the concept of self as relational as presupposed here. ${ }^{52,58)}$ Taken together, psychosis may phenomenologically be characterized by disrupted attunement which makes it impossible for the psychotic patient to relate to his environment in a subjective-experiential way. I hypothesize that such disruption in attunement may be related to the reduced impact of the external stimulus on the brain's resting state activity and hence to reduced rest-stimulus interaction in for instance the auditory cortex. My resting state hypothesis of auditory hallucinations is therefore not purely neuronal but rather neurophenomenal.

\section{CONCLUSION}

We here present a novel hypothesis about the neuronal mechanisms underlying AVH, the resting state hypothesis of AVH.

Thereby, we postulate two core assumptions which shall be briefly recounted in the following including predictions for future experimental testing. First, we hypothesize abnormally elevated resting state activity and reduced rest-stimulus interaction in auditory cortex. While elevated resting state activity in this region is supported by the data, abnormally increased rest-rest interaction remains to be demonstrated. The assumption of abnormally increased rest-rest interaction entails reduced rest-external stimulus interaction during for instance verbal stimuli which has indeed been hypothesized though not yet experimentally demonstrated. ${ }^{4)}$

Second, we hypothesize that the abnormal rest-rest interaction may be confused (or taken) by the brain with rest-external stimulus interaction thereby inferring externally located voices which in turn leads to the AVH. This inference of external voices may neurally be related to subsequent stages of auditory processing, e.g., 'upward consequences' as we called them above, which may be supported by the abnormalities in the higher cortical regions observed during AVH (see above). In terms of neural timing, one would thus expect abnormal rest-rest interaction to precede the involvement of higher cortical re- 
gions which in turn may occur prior to the onset of AVH which is at least in part in accordance with the above described findings.

In sum, we here put forward a resting state hypothesis of auditory hallucination where the resting state activity plays a central role. This does not only tell us about the pathophysiology of auditory hallucination but provides also an insight into the role of the brain's resting state in constructing phenomenal and mental features, e.g., consciousness. ${ }^{12,13)}$ Therefore I do not consider my resting state hypothesis as purely neuronal but rather as neurophenomenal for which reason I speak of a 'neurophenomenal resting state hypothesis' of auditory hallucination in schizophrenia (see title). By starting from the resting state activity and how it is related to phenomenal features of for instance auditory hallucinations, such neurophenomenal approach may provide novel insight into psychiatric symptoms including their phenomenal (i.e., subjective-experiential), nature as well as into mental features like self and consciousness in general. ${ }^{12,13)}$

\section{Acknowledgments}

Funding for this study was provided by Canadian Institute of Health Research (CIHR) and Michael Smith Foundation (EJLB-CIHR) Michael Smith Foundation, and CIHR and the EJLB Michael Smith Foundation had no further role in study design.

\section{REFERENCES}

1. Javitt DC. When doors of perception close: bottom-up models of disrupted cognition in schizophrenia. Annu Rev Clin Psychol 2009;5:249-275.

2. Langdon R, Jones SR, Connaughton E, Fernyhough C. The phenomenology of inner speech: comparison of schizophrenia patients with auditory verbal hallucinations and healthy controls. Psychol Med 2009;39:655-663.

3. Jones SR. Do we need multiple models of auditory verbal hallucinations? Examining the phenomenological fit of cognitive and neurological models. Schizophr Bull 2010;36: 566-575.

4. Allen P, Larøi F, McGuire PK, Aleman A. The hallucinating brain: a review of structural and functional neuroimaging studies of hallucinations. Neurosci Biobehav Rev 2008;32: 175-191.

5. Fletcher PC, Frith CD. Perceiving is believing: a Bayesian approach to explaining the positive symptoms of schizophrenia. Nat Rev Neurosci 2009;10:48-58.

6. Hugdahl K. "Hearing voices": auditory hallucinations as failure of top-down control of bottom-up perceptual processes. Scand J Psychol 2009;50:553-560.

7. Hoffman RE. A social deafferentation hypothesis for induction of active schizophrenia. Schizophr Bull 2007;33: 1066-1070.

8. Hoffman RE. Auditory/Verbal hallucinations, speech perception neurocircuitry, and the social deafferentation hypothesis. Clin EEG Neurosci 2008;39:87-90.

9. Buckner RL, Andrews-Hanna JR, Schacter DL. The brain's default network: anatomy, function, and relevance to disease. Ann N Y Acad Sci 2008;1124:1-38.

10. Raichle ME, MacLeod AM, Snyder AZ, Powers WJ, Gusnard DA, Shulman GL. A default mode of brain function. Proc Natl Acad Sci US A 2001;98:676-682.

11. Northoff G, Qin P, Nakao T. Rest-stimulus interaction in the brain: a review. Trends Neurosci 2010;33:277-284.

12. Northoff G. Unlocking the brain. Volume I: Coding. New York:Oxford University Press;2014.

13. Northoff G. Unlocking the brain. Volume II: Consciousness. New York:Oxford:Oxford University Press;2014.

14. Hunter MD, Eickhoff SB, Miller TW, Farrow TF, Wilkinson ID, Woodruff PW. Neural activity in speech-sensitive auditory cortex during silence. Proc Natl Acad Sci USA 2006; 103:189-194.

15. Dierks T, Linden DE, Jandl M, Formisano E, Goebel R, Lanfermann $\mathrm{H}$, et al. Activation of Heschl's gyrus during auditory hallucinations. Neuron 1999;22:615-621.

16. Diederen KM, van Lutterveld R, Sommer IE. Neuroimaging of voice hearing in non-psychotic individuals: a mini review. Front Hum Neurosci 2012;6:111.

17. Ford JM, Dierks T, Fisher DJ, Herrmann CS, Hubl D, Kindler J, et al. Neurophysiological studies of auditory verbal hallucinations. Schizophr Bull 2012;38:715-723.

18. Northoff G, Qin P. How can the brain's resting state activity generate hallucinations? A 'resting state hypothesis' of auditory verbal hallucinations. Schizophr Res 2011;127:202-214.

19. Waters F, Woodward T, Allen P, Aleman A, Sommer I. Self-recognition deficits in schizophrenia patients with auditory hallucinations: a meta-analysis of the literature. Schizophr Bull 2012;38:741-750.

20. Liddle PF. Regional brain abnormalities associated with specific syndromes of persistent schizophrenic symptoms. Clin Neuropharmacol 1992;15 Suppl 1 Pt A:401A-402A.

21. Gur RE, Mozley PD, Resnick SM, Mozley LH, Shtasel DL, Gallacher F, et al. Resting cerebral glucose metabolism in first-episode and previously treated patients with schizophrenia relates to clinical features. Arch Gen Psychiatry 1995;52:657-667.

22. Homan P, Kindler J, Hauf M, Walther S, Hubl D, Dierks T. Repeated measurements of cerebral blood flow in the left superior temporal gyrus reveal tonic hyperactivity in patients with auditory verbal hallucinations: a possible trait marker. Front Hum Neurosci 2013;7:304.

23. Diederen KM, Neggers SF, de Weijer AD, van Lutterveld $\mathrm{R}$, Daalman K, Eickhoff $\mathrm{SB}$, et al. Aberrant resting-state connectivity in non-psychotic individuals with auditory hallucinations. Psychol Med 2013;43:1685-1696.

24. Sommer IE, Clos M, Meijering AL, Diederen KM, Eickhoff SB. Resting state functional connectivity in patients with chronic hallucinations. PLoS One 2012;7:e43516.

25. Shin SE, Lee JS, Kang MH, Kim CE, Bae JN, Jung G. Segmented volumes of cerebrum and cerebellum in first episode schizophrenia with auditory hallucinations. Psychiatry Res 2005;138:33-42.

26. Wolf ND, Sambataro F, Vasic N, Frasch K, Schmid M, Schönfeldt-Lecuona $\mathrm{C}$, et al. Dysconnectivity of multiple resting-state networks in patients with schizophrenia who have persistent auditory verbal hallucinations. J Psychiatry Neurosci 2011;36:366-374.

27. Whitfield-Gabrieli S, Thermenos HW, Milanovic S, Tsuang MT, Faraone SV, McCarley RW, et al. Hyperactivity and hyperconnectivity of the default network in schizophrenia 
and in first-degree relatives of persons with schizophrenia. Proc Natl Acad Sci U S A 2009;106:1279-1284.

28. Rotarska-Jagiela A, van de Ven V, Oertel-Knöchel V, Uhlhaas PJ, Vogeley K, Linden DE. Resting-state functional network correlates of psychotic symptoms in schizophrenia. Schizophr Res 2010;117:21-30.

29. Karbasforoushan H, Woodward ND. Resting-state networks in schizophrenia. Curr Top Med Chem 2012;12:2404-2414.

30. Yu Q, Allen EA, Sui J, Arbabshirani MR, Pearlson G, Calhoun VD. Brain connectivity networks in schizophrenia underlying resting state functional magnetic resonance imaging. Curr Top Med Chem 2012;12:2415-2425.

31. Hoptman MJ, Zuo XN, Butler PD, Javitt DC, D'Angelo D, Mauro CJ, et al. Amplitude of low-frequency oscillations in schizophrenia: a resting state fMRI study. Schizophr Res 2010;117:13-20.

32. Liu H, Kaneko Y, Ouyang X, Li L, Hao Y, Chen EY, et al. Schizophrenic patients and their unaffected siblings share increased resting-state connectivity in the task-negative network but not its anticorrelated task-positive network. Schizophr Bull 2012;38:285-294.

33. Northoff G, Bermpohl F. Cortical midline structures and the self. Trends Cogn Sci 2004;8:102-107.

34. Carhart-Harris RL, Leech R, Erritzoe D, Williams TM, Stone JM, Evans J, et al. Functional connectivity measures after psilocybin inform a novel hypothesis of early psychosis. Schizophr Bull 2013;39:1343-1351.

35. Enzi B, de Greck M, Prösch U, Tempelmann C, Northoff G. Is our self nothing but reward? Neuronal overlap and distinction between reward and personal relevance and its relation to human personality. PLoS One 2009;4:e8429.

36. Northoff G, Heinzel A, de Greck M, Bermpohl F, Dobrowolny H, Panksepp J. Self-referential processing in our brain--a meta-analysis of imaging studies on the self. Neuroimage 2006;31:440-457.

37. Qin P, Northoff G. How is our self related to midline regions and the default-mode network? Neuroimage 2011; 57:1221-1233.

38. Whitfield-Gabrieli S, Moran JM, Nieto-Castañón A, Triantafyllou C, Saxe R, Gabrieli JD. Associations and dissociations between default and self-reference networks in the human brain. Neuroimage 2011;55:225-232.

39. Gawęda L, Woodward TS, Moritz S, Kokoszka A. Impaired action self-monitoring in schizophrenia patients with auditory hallucinations. Schizophr Res 2013;144:72-79.

40. Ford JM, Roach BJ, Jorgensen KW, Turner JA, Brown GG, Notestine $\mathrm{R}$, et al. Tuning in to the voices: a multisite FMRI study of auditory hallucinations. Schizophr Bull 2009;35:58-66.

41. Smith DM, Grant B, Fisher DJ, Borracci G, Labelle A, Knott VJ. Auditory verbal hallucinations in schizophrenia correlate with P50 gating. Clin Neurophysiol 2013;124: 1329-1335.

42. Shergill SS, Brammer MJ, Amaro E, Williams SC, Murray RM, McGuire PK. Temporal course of auditory hallucinations. Br J Psychiatry 2004;185:516-517.

43. Shergill SS, Bullmore E, Simmons A, Murray R, McGuire P. Functional anatomy of auditory verbal imagery in schizophrenic patients with auditory hallucinations. Am J Psychiatry 2000;157:1691-1693.

44. Hoffman RE, Anderson AW, Varanko M, Gore JC, Hampson M. Time course of regional brain activation associated with onset of auditory/verbal hallucinations. $\mathrm{Br}$
J Psychiatry 2008; 193:424-425.

45. van de Ven VG, Formisano E, Röder CH, Prvulovic D, Bittner RA, Dietz MG, et al. The spatiotemporal pattern of auditory cortical responses during verbal hallucinations. Neuroimage 2005;27:644-655.

46. Hoffman RE, Hampson M, Wu K, Anderson AW, Gore JC, Buchanan RJ, et al. Probing the pathophysiology of auditory/verbal hallucinations by combining functional magnetic resonance imaging and transcranial magnetic stimulation. Cereb Cortex 2007; 17:2733-2743.

47. Mechelli A, Allen P, Amaro E Jr, Fu CH, Williams SC, Brammer MJ, et al. Misattribution of speech and impaired connectivity in patients with auditory verbal hallucinations. Hum Brain Mapp 2007;28:1213-1222.

48. Raij TT, Valkonen-Korhonen M, Holi M, Therman S, Lehtonen J, Hari R. Reality of auditory verbal hallucinations. Brain 2009;132:2994-3001.

49. Blankenburg W. Approach to the psychopathology of common sense. Confin Psychiatr 1969;12:144-163.

50. Lysaker P, Lysaker J. Schizophrenia and the fate of the self. New York:Oxford University Press;2008.

51. Minkowski E. The essential disorder underlying schizophrenia and schizophrenic thought. In: Cutting J, Shepherd $M$, editors. The clinical roots of the schizophrenia concept: translations of seminal European contributions on schizophrenia. Orignal work published 1927. New York:Cambridge University Press; 1987.

52. Parnas J. Self and schizophrenia: A phenomenological perspective. In: Kircher T, David AS, editors. The self in neuroscience and psychiatry. New York:Cambridge University Press;2003.

53. Parnas J, Handest P. Phenomenology of anomalous self-experience in early schizophrenia. Compr Psychiatry 2003;44:121-134.

54. Parnas J, Raballo A, Handest P, Jansson L, Voller-Larsen A, Saetbye D. Self-experience in the early prodromal phases of schizophrenia. 5-year follow-up of the Copenhagen Prodromal study. World Psychiatry 2011;10:200-204.

55. Sass LA. Schizophrenia, self-experience, and the so-called 'negative symptoms'. In: Zahavi D, editor. Exploring the self: philosophical and psychopathological perspectives on self-experience. Amsterdam:John Benjamins Pub;2000.

56. Sass LA. Self-disturbance in schizophrenia: hyperreflexivity and diminished. In: Kircher T, David AS, editors. The self in neuroscience and psychiatry. New York:Cambridge University Press;2003.

57. Sass LA, Parnas J. Phenomenology of self disturbances in schizophrenia: some research findings and directions. Philos Psychiatry Psychol 2001;8:347-356.

58. Sass LA, Kircher T, David A. Self-disturbance in schizophrenia: hyperreflexivity and diminished self-affection. In: Kircher T, editor. The self in neuroscience and psychiatry. New York:Cambridge University Press;2003. No. 870539117.

59. Bin Kimura B. The self and its time (Cogito et le je). Evol psychiatr 1997;62:225-348.

60. Parnas J, Handest P, Saebye D, Jansson L. Anomalies of subjective experience in schizophrenia and psychotic bipolar illness. Acta Psychiatr Scand 2003;108:126-133.

61. Sass L. Schizophrenia, self-experience, and the so-called negative symptoms. In: Zahavi D, editor. Exploring the self: philosophical and psychopathological perspectives on selfexperience. Amsterdam:John Benjamins Pub;2000. p.149-182. 